ESAIM: PROCEEDINGS AND SURVEYS, September 2014, Vol. 45, p. 118-127

J.-S. Dhersin, Editor

\title{
PHASE-FIELD MODELS FOR THE APPROXIMATION OF THE WILLMORE FUNCTIONAL AND FLOW ${ }^{*, * *}$
}

\author{
Elie Bretin $^{1}$, Simon Masnou ${ }^{2}$ and Edouard Oudet ${ }^{3}$
}

\begin{abstract}
This paper is a short account on phase-field approximations of the Willmore functional and the associated $\mathrm{L}^{2}$-flows.
\end{abstract}

\section{INTRODUCTION}

Phase-field approximations of the Willmore functional have raised quite a lot of interest in recent years, both from the theoretical and the numerical viewpoints. In particular, attention has been paid to understanding the continuous and numerical approximations of both smooth and singular sets with finite relaxed Willmore energy. Various approximation models have been proposed so far, whose properties are known only partially. Our main motivation in this paper and in the longer version [7] is a better understanding of these models, and more precisely:

(1) Exhibiting differences/similarities between the various approximations;

(2) Deriving the $\mathrm{L}^{2}$-gradient flows associated with these models;

(3) Studying the asymptotic behavior of the flows, at least in smooth situations;

(4) Simulating numerically these flows, and observing whether and how singularities may appear.

We focus on four models due, respectively, to De Giorgi, Bellettini, and Paolini [5, 12], Bellettini [1], Mugnai [21], and Esedoḡlu, Rätz, and Röger [14].

\section{WHAT IS KNOWN?}

\subsection{De Giorgi-Bellettini-Paolini's approximation of the Willmore energy}

Based on a conjecture of De Giorgi [12], several authors have investigated the diffuse approximation of the Willmore functional, which is for a set $E \subset \mathbb{R}^{N}, N \geq 2$, with smooth boundary $\partial E$ :

$$
W(E, \Omega)=\frac{1}{2} \int_{\partial E \cap \Omega}\left|H_{\partial E}(x)\right|^{2} d \mathcal{H}^{\mathrm{N}-1}
$$

\footnotetext{
* We thank Luca Mugnai, Selim Esedoḡlu, Petru Mironescu, and Giovanni Bellettini for fruitful discussions.

** We acknowledge partial financial support from ANR (project ANR-12-BS01-0014-01 GEOMETRYA).

${ }^{1}$ Université de Lyon, CNRS UMR 5208, INSA de Lyon, Institut Camille Jordan, 20 avenue Albert Einstein, F-69621 Villeurbanne Cedex, France. Email: bretin@cmap.polytechnique.fr

2 Université de Lyon, CNRS UMR 5208, Université Lyon 1, Institut Camille Jordan, 43 boulevard du 11 novembre 1918, F-69622 Villeurbanne-Cedex, France. Email: masnou@math.univ-lyon1.fr

${ }^{3}$ Laboratoire Jean Kuntzmann, Université Joseph Fourier, Tour IRMA, BP 53, 51 rue des Mathématiques, F-38041 Grenoble Cedex 9, France. Email: edouard.oudet@imag.fr 
where $H_{\partial E}(x)$ is the classical mean curvature vector at $x \in \partial E, \mathcal{H}^{\mathrm{N}-1}$ the $(N-1)$-dimensional Hausdorff measure and $\Omega \subset \mathbb{R}^{N}$ an open set wherein the Willmore energy of $E$ is computed. The approximation functionals are defined for every $\epsilon>0$ as

$$
\mathcal{W}_{\varepsilon}(u)= \begin{cases}\frac{1}{2 \varepsilon} \int_{\Omega}\left(\varepsilon \Delta u-\frac{W^{\prime}(u)}{\varepsilon}\right)^{2} d x & \text { if } u \in \mathrm{L}^{1}(\Omega) \cap \mathrm{W}^{2,2}(\Omega) \\ +\infty & \text { otherwise in } \mathrm{L}^{1}(\Omega)\end{cases}
$$

with $W$ a double-well potential, typically $W(s)=\frac{1}{2} s^{2}(1-s)^{2}$ (other choices for $W$ are possible and yield to similar approximation results). In the sequel, we shall refer to these functionals as the classical approximation model, which was introduced by Bellettini and Paolini in [5].

The reason why $\varepsilon \Delta u-\frac{W^{\prime}(u)}{\varepsilon}$ is related to mean curvature can be simply understood at a formal level: it suffices to observe that the mean curvature of a smooth surface is associated with the first variation of its area, and that $-\varepsilon \Delta u+\frac{W^{\prime}(u)}{\varepsilon}$ is the $\mathrm{L}^{2}$-gradient of $\frac{\varepsilon}{2}|\nabla u|^{2}+\frac{W(u)}{\varepsilon}$ that appears in the approximation of surface area.

The results on the asymptotic behavior of $\mathcal{W}_{\varepsilon}$ as $\varepsilon \rightarrow 0^{+}$started with the proof by Bellettini and Paolini [5] of a $\Gamma$ - limsup property, i.e. the Willmore energy of a smooth hypersurface $E$ is the limit of $\mathcal{W}_{\varepsilon}\left(u_{\varepsilon}\right)$, up to a multiplicative constant, where $u_{\varepsilon}$ is defined exactly as for the approximation of the perimeter.

The $\Gamma$ - lim inf property is much harder to prove. The contributions on this point $[3,20,22,24,26]$ culminated with the proof by Röger and Schätzle [24] in space dimensions $N=2,3$ and, independently, by Nagase and Tonegawa [22] in dimension $N=2$, that the result holds true for smooth sets. More precisely, given $u=1_{E}$ the characteristic function of a set $E \in \mathrm{C}^{2}(\Omega)$, and $u_{\varepsilon}$ converging to $u$ in $\mathrm{L}^{1}(\Omega)$ with a uniform control of the approximating perimeter $P_{\varepsilon}\left(u_{\varepsilon}\right)$, then $c_{0} W(E, \Omega) \leq \liminf _{\varepsilon \rightarrow 0^{+}} \mathcal{W}_{\varepsilon}\left(u_{\varepsilon}\right)$.

What about unsmooth sets? Can the approximation results be extended to the relaxed Willmore functional? The answer is negative in general, as discussed below.

\subsection{The approximation does not hold in general for unsmooth limit sets}

Define for any set $E$ of finite perimeter in $\Omega$ its relaxed Willmore functional

$$
\bar{W}(E, \Omega)=\inf \left\{\liminf W\left(E_{h}, \Omega\right), \partial E_{h} \cap \Omega \in \mathrm{C}^{2}, E_{h} \rightarrow E \text { in } \mathrm{L}^{1}(\Omega)\right\} .
$$

It is natural to ask whether the $\Gamma$-convergence of $\mathcal{W}_{\varepsilon}$ to $W$ can be extended to $\bar{W}$. Unfortunately, this is not the case as it follows from the following observations (for simplicity we denote $\Gamma-\lim \mathcal{W}_{\varepsilon}(E)=\Gamma-\lim \mathcal{W}_{\varepsilon}\left(1_{E}\right)$ ) that are illustrated in Figure 1:

(1) there exists a bounded set $E_{1} \subset \mathbb{R}^{2}$ of finite perimeter such that $\Gamma-\lim \mathcal{W}_{\varepsilon}\left(E_{1}\right)<\infty$ and $\bar{W}\left(E_{1}\right)=+\infty$;

(2) there exists a bounded set $E_{2} \subset \mathbb{R}^{2}$ of finite perimeter such that $\Gamma-\lim \mathcal{W}_{\varepsilon}\left(E_{2}\right)<\bar{W}\left(E_{2}\right)<+\infty$.
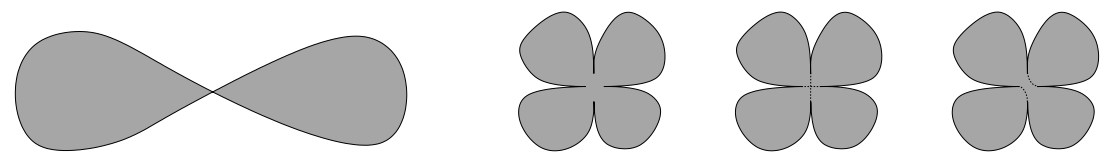

FiguRE 1. Left: a set $E_{1}$ such that $\Gamma-\lim \mathcal{W}_{\varepsilon}\left(E_{1}\right)<\infty$ and $\bar{W}\left(E_{1}\right)=+\infty$. Then, from left to right, a set $E_{2}$, the limit configuration whose energy coincides with $\Gamma-\lim \mathcal{W}_{\varepsilon}\left(E_{2}\right)$, and a configuration whose energy coincides with $\bar{W}\left(E_{2}\right)$.

The reason why $\bar{W}\left(E_{1}\right)=+\infty$ is a result by Bellettini, Dal Maso, and Paolini [2] according to which a non oriented tangent must exist everywhere on the boundary. Besides, $\bar{W}\left(E_{2}\right)<+\infty$ because, still by a result of Bellettini, Dal Maso and Paolini, the boundary is smooth out of evenly many cusps. Let us now explain why, in both cases, $\Gamma-\lim \mathcal{W}_{\varepsilon}\left(E_{1,2}\right)<+\infty$. The reason for this is the existence of smooth solutions with singular 
nodal sets for the Allen-Cahn equation $\Delta u-W^{\prime}(u)=0$. According to Dang, Fife and Peletier [11], there exists for such equation in $\mathbb{R}^{2}$ a unique saddle solution $u$ with values in $(-1,1)$. By saddle solution, it is meant that $u(x, y)>0$ in quadrants I and III, and $u(x, y)<0$ in quadrants II and IV, in particular $u(x, y)=0$ on the nodal set $x y=0$. Considering $u_{\varepsilon}(x)=u(\varepsilon x)$, we immediately get that $\varepsilon^{2} \Delta u_{\varepsilon}-W^{\prime}\left(u_{\varepsilon}\right)=0$, thus the second term in $\mathcal{W}_{\varepsilon}\left(u_{\varepsilon}\right)$ vanishes, and the first term being obviously bounded, it follows from the lower semicontinuity of the $\Gamma$-limit that $\Gamma-\lim \mathcal{W}_{\varepsilon}\left(E_{1,2}\right)<+\infty$. Furthermore, the approximation of $E_{2}$ can be made so as to create a cross in the limit, as in bottom-middle figure. The limit energy is therefore lower than the energy obtained by pairwise connection without crossing of the cusps (bottom-right figure). Thus, $\Gamma-\lim \mathcal{W}_{\varepsilon}\left(E_{2}\right)<\bar{W}\left(E_{2}\right)<+\infty$. We end this section with the question that follows naturally from the discussion above: is it possible to find a diffuse approximation that $\Gamma$-converges to $\bar{W}$ (up to a multiplicative constant) whenever $\bar{W}(E)<+\infty$ ?

\subsection{Diffuse approximations of the relaxed Willmore functional}

\subsubsection{Bellettini's approximation in dimension $N \geq 2$}

In [1], Bellettini proposed a diffuse model for approximating the relaxations of geometric functionals of the form $\int_{\partial E}\left(1+f\left(x, \nabla d_{E}, D^{2} d_{E}\right)\right) d \mathcal{H}^{\mathrm{N}-1}$ where $E$ is smooth and $d_{E}$ is the signed distance function from $\partial E$. Particularizing Bellettini's approximation model to the Willmore energy yields the smooth functionals

$$
\mathcal{W}_{\varepsilon}^{\mathrm{Be}}(u)= \begin{cases}\frac{1}{2} \int_{\Omega \backslash\{|\nabla u|=0\}}\left(\left|\operatorname{div} \frac{\nabla u}{|\nabla u|}\right|^{2}\right)\left(\frac{\varepsilon}{2}|\nabla u|^{2}+\frac{W(u)}{\varepsilon}\right) d x & \text { if } u \in \mathrm{C}^{2}(\Omega) \cap \mathrm{L}^{1}(\Omega) \\ +\infty & \text { otherwise in } \mathrm{L}^{1}(\Omega)\end{cases}
$$

Then, according to Bellettini [1, Thms 4.2,4.3], in any space dimension $N \geq 2$,

$$
\left(\Gamma-\lim _{\varepsilon \rightarrow 0} P_{\varepsilon}+\mathcal{W}_{\varepsilon}^{\mathrm{Be}}\right)(E)=c_{0}(P(E)+\bar{W}(E)) \quad \text { for every } E \text { of finite perimeter such that } \bar{W}(E)<+\infty .
$$

\subsubsection{Mugnai's approximation in dimension $N=2$}

In the regular case and in dimensions 2,3, it follows from the results of Bellettini and Mugnai [4] that, up to a uniform control of the perimeter, the $\Gamma$-limit of the functionals defined by

$$
\mathcal{W}_{\varepsilon}^{\mathrm{Mu}}(u)= \begin{cases}\frac{1}{2 \varepsilon} \int_{\Omega}\left|\varepsilon D^{2} u-\frac{W^{\prime}(u)}{\varepsilon} \nu_{u} \otimes \nu_{u}\right|^{2} d x & \text { if } u \in \mathrm{C}^{2}(\Omega) \cap \mathrm{L}^{1}(\Omega) \\ +\infty & \text { otherwise in } \mathrm{L}^{1}(\Omega)\end{cases}
$$

where $\nu_{u}=\frac{\nabla u}{|\nabla u|}$ when $|\nabla u| \neq 0$, and $\nu_{u}=a$ constant unit vector on $\{|\nabla u|=0\}$, coincides with $c_{0} \int_{\Omega \cap \partial E}\left|A_{\partial E}(x)\right|^{2} d x$ for every smooth $E$, with $A_{\partial E}(x)$ the second fundamental form of $\partial E$ at $x$. In dimension 2, the second fundamental form along a curve coincides with the curvature. Therefore, by identifying the limit varifold obtained when $u_{\varepsilon}$ converges to $u=1_{E}$, Mugnai was able to prove in [21] that, in dimension 2, the $\Gamma$-limit of $\mathcal{W}_{\varepsilon}^{\mathrm{Mu}}$ (with uniform control of the perimeter) coincides with $\bar{W}(E)$ for any $E$ with finite perimeter, up to a multiplicative constant.

\subsubsection{Esedoḡlu-Rätz-Röger's approximation in dimension $N \geq 2$}

The model of Esedoglu, Rätz, and Röger in [14] is a modification of the classical energy that aims to preserve the "parallelism" of the level lines of the approximating functions, and avoids the formation of saddle points, by constraining the level lines' mean curvature using a term à la Bellettini. More precisely, a natural profile-forcing approximation model is (with $\alpha \geq 0$ a parameter):

$\mathcal{W}_{\varepsilon}^{\text {EsRäRö }}(u)=\left\{\begin{array}{l}\frac{1}{2 \varepsilon} \int_{\Omega}\left(\varepsilon \Delta u-\frac{W^{\prime}(u)}{\varepsilon}\right)^{2} d x+\frac{1}{2 \varepsilon^{1+\alpha}} \int_{\Omega}\left(\varepsilon \Delta u-\frac{W^{\prime}(u)}{\varepsilon}-\varepsilon|\nabla u| \operatorname{div} \frac{\nabla u}{|\nabla u|}\right)^{2} d x \quad \text { if } u \in \mathrm{C}^{2}(\Omega) \cap \mathrm{L}^{1}(\Omega) \\ +\infty \\ \text { otherwise in } L^{1}(\Omega)\end{array}\right.$ 
To simplify the theoretical analysis, the model proposed by Esedoḡlu, Rätz, and Röger is slightly different:

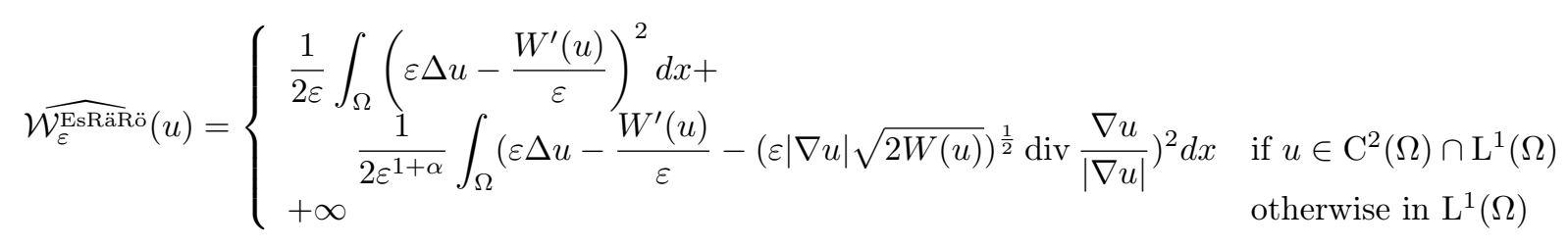

Esedoḡlu, Rätz, and Röger prove that, for any $\alpha>0, \Gamma-\lim _{\varepsilon \rightarrow 0} P_{\varepsilon}+\widehat{\mathcal{W}_{\varepsilon}^{\text {EsRär }} \text { ö }}=c_{0}(P+\bar{W})$ in $\mathrm{L}^{1}(\Omega)$. With $\alpha=0$ the $\Gamma$-convergence result does not hold anymore, but instead, with a uniform control of the perimeter, one has $\Gamma-\lim _{\varepsilon \rightarrow 0} \widehat{\mathcal{W}} \widehat{\underline{\varepsilon} \text { ERäRö }} \geq \frac{c_{0}}{2} \bar{W}$, which still guarantees a control of $\bar{W}$.

\section{The Willmore flow and its approximation by the Evolution of a Diffuse INTERFACE}

This section is devoted to the approximation of the Willmore flow by $\mathrm{L}^{2}$-gradient flows associated with the approximating energies introduced above. In particular, we shall derive explicitly each approximating gradient flow and, using the matched asymptotic expansion method $[6,8,18,23]$, we will show that, at least formally and for smooth interfaces, there is convergence to the Willmore flow, at least in dimensions 2 and 3 for all flows, and in any dimension for some of them. The general question "if a sequence of functionals $\Gamma$-converges to a limit functional, is there also convergence of the associated flows?" is rather natural, since $\Gamma$-convergence implies convergence of minimizers, up to the extraction of a subsequence. However, the question is difficult and remains open for the Willmore functional. Our results below give formal indications that the convergence holds. Serfaty discussed in [25] a general theorem on the $\Gamma$-convergence of gradient flows, provided that the generalized gradient of the associated functional can be controlled (see in particular the discussion on the Cahn-Hilliard flow). Such control is so far out of reach for the Willmore functional.

\subsection{On the Willmore flow}

Let $E(t), 0 \leq t \leq T$, represent the evolution by the Willmore flow of smooth domains, i.e. the outer normal velocity field $V(t)$ is given at $x \in \partial E(t)$ by $V=\Delta_{\partial E(t)} H-\frac{1}{2} H^{3}+H\|A\|^{2}$, where $\Delta_{\partial E(t)}$ is the Laplace-Beltrami operator on $\partial E(t), H$ the scalar mean curvature, $A$ the second fundamental form, and $\|A\|^{2}$ is the sum of the squared coefficients of $A$.

In the plane, the Willmore flow coincides with the flow of curves associated with the Bernoulli-Euler elastica energy, i.e., denoting by $\kappa$ the scalar curvature, $V=\Delta_{\partial E(t)} \kappa+\frac{1}{2} \kappa^{3}$. The long time existence of a single curve evolving by this flow is established in [13], and any curve with fixed length converges to an elastica.

In higher dimension, Kuwert and Schätzle give in $[15,16]$ a long time existence proof of the Willmore flow and the convergence to a round sphere for sufficiently small initial energy. Singularities may appear for larger initial energies, as indicated by numerical simulations [19].

\subsection{Approximating the Willmore flow with the classical De Giorgi-Bellettini-Paolini's approach}

The $\mathrm{L}^{2}$-gradient flow of the approximating energy $\mathcal{W}_{\varepsilon}(u)=\frac{1}{2 \varepsilon} \int_{\Omega}\left(\varepsilon \Delta u-\frac{W^{\prime}(u)}{\varepsilon}\right)^{2} d x$, is equivalent to the evolution equation $\partial_{t} u=-\Delta\left(\Delta u-\frac{1}{\varepsilon^{2}} W^{\prime}(u)\right)+\frac{1}{\varepsilon^{2}} W^{\prime \prime}(u)\left(\Delta u-\frac{1}{\varepsilon^{2}} W^{\prime}(u)\right)$, that can be rewritten as the phase field system

$$
\left\{\begin{array}{l}
\varepsilon^{2} \partial_{t} u=\Delta \mu-\frac{1}{\varepsilon^{2}} W^{\prime \prime}(u) \mu \\
\mu=W^{\prime}(u)-\varepsilon^{2} \Delta u .
\end{array}\right.
$$


The well-posedness of the phase-field model (1) at fixed parameter $\varepsilon$ has been studied in [9] with a volume constraint fixing the average of $u$, and in [10] with both volume and area constraints. Loreti and March showed in [18], using the formal method of matched asymptotic expansions, that if $\partial E$ is smooth and evolves by Willmore flow, it can be approximated by level lines of the solution $u_{\varepsilon}$ to the phase field system (1) as $\varepsilon$ goes to 0 . In addition, $u_{\varepsilon}$ and $\mu_{\varepsilon}$ are expected to take the form $u_{\varepsilon}(x, t)=q\left(\frac{d(x, E(t))}{\varepsilon}\right)+\varepsilon^{2}\left(\|A\|^{2}-\frac{1}{2} H^{2}\right) \eta_{1}\left(\frac{d(x, E(t))}{\varepsilon}\right)+O\left(\varepsilon^{3}\right)$ and $\mu_{\varepsilon}(x, t)=-\varepsilon H q^{\prime}\left(\frac{d(x, E(t))}{\varepsilon}\right)+\varepsilon^{2} H^{2} \eta_{2}\left(\frac{d(x, E(t))}{\varepsilon}\right)+O\left(\varepsilon^{3}\right)$, where $\eta_{1}$ and $\eta_{2}$ are two functions depending only on the double well potential $W$. An important point is that the second-order term in the asymptotic expansion of $u_{\varepsilon}$ has an influence on the limit law as $\varepsilon$ goes to zero [18]. This is a major difference with the Allen-Cahn equation, for which the velocity law follows from the expansion at zero and first orders only [6]. As a consequence, addressing numerically the Willmore flow is more delicate and requires using a high accuracy approximation in space to guarantee a sufficiently good approximation of the expansion of $u_{\varepsilon}$.

\subsection{Approximating the Willmore flow with Bellettini's model}

We now focus on the approximation model $\mathcal{W}_{\varepsilon}^{\mathrm{Be}}(u)=\frac{1}{2} \int_{\Omega} \operatorname{div}\left(\frac{\nabla u}{|\nabla u|}\right)^{2}\left(\frac{\varepsilon}{2}|\nabla u|^{2}+\frac{1}{\varepsilon} W(u)\right) d x$.

Proposition 3.1. The $\mathrm{L}^{2}$-gradient flow of Bellettini's model is equivalent to

$$
\partial_{t} u=\frac{K(u)^{2}}{2}\left(\Delta u-\frac{1}{\varepsilon^{2}} W^{\prime}(u)\right)+\frac{1}{2} \nabla\left[K(u)^{2}\right] . \nabla u-\frac{1}{\varepsilon} \operatorname{div}\left(P^{u} \frac{\nabla\left[K(u) h_{\varepsilon}(u)\right]}{|\nabla u|}\right),
$$

where $P^{u}=I_{d}-\frac{\nabla u}{|\nabla u|} \otimes \frac{\nabla u}{|\nabla u|}, h_{\varepsilon}(u)=\left(\frac{\varepsilon}{2}|\nabla u|^{2}+\frac{1}{\varepsilon} W(u)\right)$ and $K(u)=\operatorname{div}\left(\frac{\nabla u}{|\nabla u|}\right)$.

Proof. See [7]

Claim 3.2. In a suitable regime provided by the method of matched asymptotic expansions, the normal velocity of the $\frac{1}{2}$-front $\Gamma(t)=\partial E(t)$ associated with a solution $u_{\varepsilon}(x, t)$ to Bellettini's phase field model (2) is the Willmore velocity $V=\Delta_{\Gamma} H+\|A\|^{2} H-\frac{H^{3}}{2}$, and one has $u_{\varepsilon}(x, t)=q\left(\frac{d(x, E(t))}{\varepsilon}\right)+O\left(\varepsilon^{2}\right)$.

Proof. See [7]. The asymptotic derivation shows in particular that the second-order term in $u_{\varepsilon}(x, t)$ does not appear in the expression of $V$. This explains the numerical stability, despite the use of an explicit Euler scheme, observed by Esedoğlu, Rätz, and Röger in [14].

\subsection{Approximating the Willmore flow with Mugnai's model}

Proposition 3.3. The $\mathrm{L}^{2}$-gradient flow of Mugnai's model is equivalent to

$$
\left\{\begin{array}{l}
\varepsilon^{2} \partial_{t} u=\Delta \mu-\frac{1}{\varepsilon^{2}} W^{\prime \prime}(u) \mu+W^{\prime}(u)\left(\operatorname{div}\left(\operatorname{div}\left(\frac{\nabla u}{|\nabla u|}\right) \frac{\nabla u}{|\nabla u|}\right)-\operatorname{div}\left(D\left(\frac{\nabla u}{|\nabla u|}\right) \frac{\nabla u}{|\nabla u|}\right)\right. \\
\mu=\frac{1}{\varepsilon^{2}} W^{\prime}(u)-\Delta u
\end{array}\right.
$$

Proof. See [7]. Note that this system coincides with the classical one, up to the addition of a penalty term.

Claim 3.4. In a suitable regime provided by the method of matched asymptotic expansions, the normal velocity of the $\frac{1}{2}$-front $\Gamma(t)=\partial E(t)$ associated with a solution $\left(u_{\varepsilon}, \mu_{\varepsilon}\right)$ to Mugnai's phase field model (3) is $V=\Delta_{\Gamma} H+\sum_{i} \kappa_{i}^{3}-\frac{1}{2}\|A\|^{2} H$. Moreover, $\left\{\begin{array}{l}u_{\varepsilon}(x, t)=q\left(\frac{d(x, E(t))}{\varepsilon}\right)+\varepsilon^{2} \frac{\|A\|^{2}}{2} \eta_{1}\left(\frac{d(x, E(t)}{\varepsilon}\right)+O\left(\varepsilon^{3}\right) \\ \mu_{\varepsilon}(x, t)=-\varepsilon H q^{\prime}\left(\frac{d(x, E(t)}{\varepsilon}\right)+\|A\|^{2} \varepsilon^{2} \eta_{2}\left(\frac{d(x, E(t)}{\varepsilon}\right)+O\left(\varepsilon^{3}\right)\end{array}\right.$, where $\eta_{1}$ and $\eta_{2}$ are profile functions.

Proof. See [7]. 
Remark 3.5. The front velocity associated with Mugnai's phase field model coincides, up to a multiplicative constant, with the velocity of the $\mathrm{L}^{2}$-flow of the squared second fundamental form energy $\int_{\Gamma}\|A\|^{2} d \mathcal{H}^{\mathrm{N}-1}$. In dimensions 2 and 3, it is easily seen that Mugnai's flow coincides with the Willmore flow.

\subsection{Approximating the Willmore flow with Esedoglu-Rätz-Röger's energy}

We now consider the following variant of the Esedoğlu-Rätz-Röger's energy,

$$
\mathcal{W}_{\varepsilon}^{\text {EsRäRö }}(u)=\frac{1}{2 \varepsilon} \int_{\Omega}\left(\varepsilon \Delta u-\frac{W^{\prime}(u)}{\varepsilon}\right)^{2} d x+\frac{\beta}{\varepsilon^{1+\alpha}} \int_{\Omega}\left(\varepsilon D^{2} u: \frac{\nabla u}{|\nabla u|} \otimes \frac{\nabla u}{|\nabla u|}-\frac{W^{\prime}(u)}{\varepsilon}\right)^{2} d x
$$

Proposition 3.6. The $\mathrm{L}^{2}$-gradient flow of Esedoḡlu-Rätz-Röger's model is equivalent to

$$
\left\{\begin{array}{l}
\varepsilon^{2} \partial_{t} u=\Delta \mu-\frac{1}{\varepsilon^{2}} W^{\prime \prime}(u) \mu-\beta \widetilde{L}(u) \\
\mu=W^{\prime}(u)-\varepsilon^{2} \Delta u . \\
\xi_{\varepsilon}=\varepsilon D^{2} u: \frac{\nabla u}{|\nabla u|} \otimes \frac{\nabla u}{|\nabla u|}-\frac{W^{\prime}(u)}{\varepsilon} \\
\widetilde{L}(u)=2 \varepsilon^{1-\alpha}\left[\left(\frac{\nabla u}{|\nabla u|} \otimes \frac{\nabla u}{|\nabla u|}: D^{2} \xi_{\varepsilon}-\frac{1}{\varepsilon^{2}} W^{\prime \prime}(u) \xi_{\varepsilon}\right)+2\left(\operatorname{div}\left(\frac{\nabla u}{|\nabla u|}\right) \frac{\nabla u}{|\nabla u|}\right) \cdot \nabla \xi_{\varepsilon}+\mathcal{B}(u) \xi_{\varepsilon}\right] \\
\mathcal{B}(u)=\operatorname{div}\left(\operatorname{div}\left(\frac{\nabla u}{|\nabla u|}\right) \frac{\nabla u}{|\nabla u|}\right)-\operatorname{div}\left(D\left(\frac{\nabla u}{|\nabla u|}\right) \frac{\nabla u}{|\nabla u|}\right)
\end{array}\right.
$$

Proof. See [7]. Note that this system coincides with the classical one, up to the addition of a penalty term.

Claim 3.7. In a suitable regime provided by the method of matched asymptotic expansions, the normal velocity of the $\frac{1}{2}$-front $\Gamma(t)=\partial E(t)$ associated with a solution $\left(u_{\varepsilon}^{\alpha}, \mu_{\varepsilon}^{\alpha}, \xi_{\varepsilon}^{\alpha}\right)$ to Esedoglu-Rätz-Röger's phase field model (4) in both cases $\alpha=0$ and $\alpha=1$ is the Willmore velocity $V=\Delta_{\Gamma} H+\|A\|^{2} H-\frac{H^{3}}{2}$. In addition, for $\alpha=0$ :

$$
\left\{\begin{array}{l}
u_{\varepsilon}^{0}(x, t)=q\left(\frac{d(x, E(t))}{\varepsilon}\right)+\varepsilon^{2} \frac{\|A\|^{2}-H^{2}}{1+2 \beta} \eta_{1}\left(\frac{d(x, E(t)}{\varepsilon}\right)+O\left(\varepsilon^{3}\right) \\
\mu_{\varepsilon}^{0}(x, t)=-\varepsilon H q^{\prime}\left(\frac{d(x, E(t)}{\varepsilon}\right)+\varepsilon^{2}\left(H^{2}-2 \beta\left[\frac{2\|A\|^{2}-H^{2}}{1+2 \beta}\right]\right) \eta_{2}\left(\frac{d(x, E(t)}{\varepsilon}\right)+O\left(\varepsilon^{3}\right), \\
\xi_{\varepsilon}^{0}(x, t)=\varepsilon\left(\frac{2\|A\|^{2}-H^{2}}{1+2 \beta}\right) \eta_{2}\left(\frac{d(x, E(t)}{\varepsilon}\right)+O\left(\varepsilon^{2}\right)
\end{array}\right.
$$

where $\eta_{2}(z)=z q^{\prime}(z)$ is a profile function. For $\alpha=1,\left\{\begin{array}{l}u_{\varepsilon}^{1}(x, t)=q\left(\frac{d(x, E(t))}{\varepsilon}\right)+O\left(\varepsilon^{3}\right) \\ \mu_{\varepsilon}^{1}(x, t)=-\varepsilon H q^{\prime}\left(\frac{d(x, E(t)}{\varepsilon}\right)+2 \varepsilon^{2}\|A\|^{2} \eta_{2}\left(\frac{d(x, E(t)}{\varepsilon}\right)+O\left(\varepsilon^{3}\right), \\ \xi_{\varepsilon}^{1}(x, t)=\varepsilon^{2} \frac{\left(2\|A\|^{2}-H^{2}\right)}{4 \beta} \eta_{2}\left(\frac{d(x, E(t)}{\varepsilon}\right)+O\left(\varepsilon^{3}\right)\end{array}\right.$

Remark 3.8. The previous claim gives indications on the design of a numerical scheme for simulating the Esedoglu-Rätz-Röger's flow in the cases $\alpha=0,1$. Clearly, the flow acts at the second order for $u$ in the case $\alpha=0$, and not less than at the third order (at least) whenever $\alpha=1$. This implies that capturing with accuracy the motion of the interface should be much more delicate when $\alpha=1$. The analysis of the asymptotic behavior for $\alpha$ non integer is more delicate because it requires studying non integer orders of $\varepsilon$ and it is far from being clear how integer and non integer scales may combine. As for integer values of $\alpha>1$, a careful study at higher orders of $\varepsilon$ should be possible but is out of the scope of the present paper.

\section{Numerical Simulations for the Classical And Mugnai's Diffuse flows}

In this paper, we consider a new scheme, quite different from what is usually done in the literature, to solve both the classical and Mugnai's phase field systems (1) and (3). The simulations can be compared with those obtained by Esedoğlu, Rätz, and Röger in [14] for their phase field system (4) (actually a variant of it, see [14]), and for Bellettini's phase field system (2). 


\subsection{New numerical schemes for the approximation of the classical and Mugnai's flows}

\subsubsection{Classical diffuse approximation flow}

We introduce a new scheme to approximate numerically some solutions of the phase field system

$$
\left\{\begin{array}{l}
\partial_{t} u=\frac{1}{\alpha \varepsilon^{2}} \Delta \mu-\frac{1}{\alpha \varepsilon^{4}} W^{\prime \prime}(u) \mu \\
\mu=\alpha W^{\prime}(u)-\alpha \varepsilon^{2} \Delta u
\end{array}\right.
$$

where $\alpha$ is a positive constant. The particular phase field system (1) corresponding to the classical diffuse Willmore flow is obtained for $\alpha=1$. We compute the solution for any time $t \in[0, T]$ in a box $\Omega=[0,1]^{N}$ with periodic boundary conditions. We use an Euler implicit discretization in time:

$$
\left\{\begin{array}{l}
u^{n+1}=\delta_{t}\left[\frac{1}{\alpha \varepsilon^{2}} \Delta \mu^{n+1}-\frac{1}{\alpha \varepsilon^{4}} W^{\prime \prime}\left(u^{n+1}\right) \mu^{n+1}\right]+u^{n} \\
\mu^{n+1}=\alpha W^{\prime}\left(u^{n+1}\right)-\alpha \varepsilon^{2} \Delta u^{n+1}
\end{array}\right.
$$

where $\delta_{t}$ is the time step, $u^{n}$ and $\mu^{n}$ are the approximations of the solutions $u$ and $\mu$, respectively, evaluated at time $t_{n}=n \delta_{t}$. The system can be written as $\left\{\begin{array}{l}u^{n+1}-\frac{\delta_{t}}{\alpha \varepsilon^{2}} \Delta \mu^{n+1}=E \\ \mu^{n+1}+\alpha \varepsilon^{2} \Delta u^{n+1}=F,\end{array}\right.$ with $E=u^{n}-\frac{\delta_{t}}{\alpha \varepsilon^{4}} W^{\prime \prime}\left(u^{n+1}\right) \mu^{n+1}$, $F=\alpha W^{\prime}\left(u^{n+1}\right)$. Thus, $\left(u^{n+1}, \mu^{n+1}\right)$ is the solution of the nonlinear equation $\left(\begin{array}{l}u^{n+1} \\ \mu^{n+1}\end{array}\right)=\phi\left(\begin{array}{l}u^{n+1} \\ \mu^{n+1}\end{array}\right)$, where

$$
\phi\left(\begin{array}{c}
u^{n+1} \\
\mu^{n+1}
\end{array}\right)=\left(I_{d}+\delta_{t} \Delta^{2}\right)^{-1}\left(\begin{array}{cc}
I_{d} & \frac{\delta_{t}}{\alpha \varepsilon^{2}} \Delta \\
-\alpha \varepsilon^{2} \Delta & I_{d}
\end{array}\right)\left(\begin{array}{c}
u^{n}-\frac{\delta_{t}}{\alpha \varepsilon^{4}} W^{\prime \prime}\left(u^{n+1}\right) \mu^{n+1} \\
\alpha W^{\prime}\left(u^{n+1}\right)
\end{array}\right)
$$

A natural way to approximate $\left(u^{n+1}, \mu^{n+1}\right)$ is a fixed point iterative method:

Algorithm 4.1. Initialization : $u_{0}^{n+1}=u^{n}, \mu_{0}^{n+1}=\mu_{n}$ While $\left\|u_{k+1}^{n+1}-u_{k}^{n+1}\right\|+\left\|\mu_{k+1}^{n+1}-\mu_{k}^{n+1}\right\|>10^{-8}$, perform the loop on $k$ :

1) Compute $h_{\mathcal{P}}^{n}=u^{n}-\frac{\delta_{t}}{\alpha \varepsilon^{4}} W^{\prime \prime}\left(u_{k}^{n+1}\right) \mu_{k}^{n+1}$ and $\tilde{h}_{\mathcal{P}}^{n}=\alpha W^{\prime}\left(u_{k}^{n+1}\right)$.

2) Using the Fast Fourier Transform, compute the truncated Fourier series of $h_{\mathcal{P}}^{n}$ and $\tilde{h}_{\mathcal{P}}^{n}$ :

$$
h_{\mathcal{P}}^{n}(x)=\sum_{\|p\|_{\infty} \leq \mathcal{P}} h_{p}^{n} e^{2 i \pi x \cdot p}, \quad \text { and } \quad \tilde{h}_{\mathcal{P}}^{n}(x)=\sum_{\|p\|_{\infty} \leq \mathcal{P}} \tilde{h}_{p}^{n} e^{2 i \pi x \cdot p}
$$

3) Compute $u_{k+1}^{n+1}(x)=\sum_{\|p\|_{\infty} \leq \mathcal{P}}\left(u_{k+1}\right)_{p}^{n} e^{2 i \pi x \cdot p}$ and $\mu_{k+1}^{n+1}(x)=\sum_{\|p\|_{\infty} \leq \mathcal{P}}\left(\mu_{k+1}\right)_{p}^{n} e^{2 i \pi x \cdot p}$, where

$$
\left\{\begin{array}{l}
\left(u_{k+1}\right)_{p}^{n}=\frac{1}{1+\delta_{t}\left(4 \pi^{2}|p|\right)^{2}}\left(h_{p}^{n}-\frac{\delta_{t}}{\alpha \varepsilon^{2}} 4 \pi^{2}|p|^{2} \tilde{h}_{p}^{n}\right) \\
\left(\mu_{k+1}\right)_{p}^{n}=\frac{1}{1+\delta_{t}\left(4 \pi^{2}|p|\right)^{2}}\left(\tilde{h}_{p}^{n}+\alpha \varepsilon^{2} 4 \pi^{2}|p|^{2} h_{p}^{n}\right)
\end{array}\right.
$$

End

Proposition 4.2. Algorithm 4.1 converges locally under the following assumption, where $M_{i}=\sup _{s \in[0,1]}\left|W^{(i)}(s)\right|$ :

$$
\max \left\{\left(\alpha M_{2}\right)^{2}+2\left(\frac{\delta_{t}}{\varepsilon^{4}} M_{3}\left(M_{1}+N^{3 / 2} \pi^{2} \frac{\varepsilon^{2}}{\delta_{x}^{5 / 2}}\right)\right)^{2}, 2\left(\frac{\delta_{t}}{\alpha \varepsilon^{4}} M_{2}\right)^{2}\right\}<1 .
$$

Proof. See [7]. 


\subsubsection{Mugnai's flow}

We now use a similar scheme for the following generalization of Mugnai's phase field system:

$$
\left\{\begin{array}{l}
\partial_{t} u=\frac{1}{\varepsilon^{2} \alpha} \Delta \mu-\frac{1}{\varepsilon^{4} \alpha} W^{\prime \prime}(u) \mu+\widetilde{\mathcal{B}}(u) \\
\mu=\alpha W^{\prime}(u)-\alpha \varepsilon^{2} \Delta u
\end{array}\right.
$$

with $\widetilde{\mathcal{B}}(u)=\frac{W^{\prime}(u) \mathcal{B}(u)}{\alpha \varepsilon^{4}}$. The exact Mugnai's flow (3) corresponds to the choice $\alpha=1$ (up to a time rescaling). We use an Euler semi-implicit discretization in time:

$$
\left\{\begin{array}{l}
u^{n+1}=\delta_{t}\left[\frac{1}{\varepsilon^{2} \alpha} \Delta \mu^{n+1}-\frac{1}{\alpha \varepsilon^{4}} W^{\prime \prime}\left(u^{n+1}\right) \mu^{n+1}+\widetilde{\mathcal{B}}\left(u^{n}\right)\right]+u^{n} \\
\mu^{n+1}=\alpha W^{\prime}\left(u^{n+1}\right)-\varepsilon^{2} \alpha \Delta u^{n+1}
\end{array}\right.
$$

We use a fixed point iteration to approximate the solution pair $\left(u^{n+1}, \mu^{n+1}\right)$ to the system:

$$
\left(u^{n+1}, \mu^{n+1}\right)=\tilde{\phi}\left(u^{n+1}, \mu^{n+1}\right)=\left(I_{d}+\delta_{t} \Delta^{2}\right)^{-1}\left(\begin{array}{cc}
I_{d} & \frac{\delta_{t}}{\alpha \varepsilon^{2}} \Delta \\
-\alpha \varepsilon^{2} \Delta & I_{d}
\end{array}\right)\left(\begin{array}{c}
u^{n}-\frac{\delta_{t}}{\alpha \varepsilon^{4}} W^{\prime \prime}\left(u^{n+1}\right) \mu^{n+1}+\delta_{t} \widetilde{\mathcal{B}}\left(u^{n}\right) \\
\alpha W^{\prime}\left(u^{n+1}\right)
\end{array}\right) .
$$

For it is highly singular, the penalization term $\widetilde{\mathcal{B}}(\cdot)$ needs to be regularized to avoid numerical errors. We use instead the regularized penalization term $\widetilde{\mathcal{B}}_{\sigma}(u)=W^{\prime}(u)\left[\left(\left|\nabla \nu_{u, \sigma}\right|^{2}-\left|\operatorname{div} \nu_{u, \sigma}\right|^{2}\right)-\operatorname{curl}\left(\operatorname{curl}\left(\nu_{u, \sigma}\right)\right) \cdot \nu_{u, \sigma}\right] \operatorname{where}$ $\nu_{u, \sigma}=\frac{\nabla u}{\sqrt{|\nabla u|^{2}+\sigma^{2}}}$ with $\sigma$ a small regularization parameter. In particular, the positivity of $\left(\left|\nabla \nu_{u, \sigma}\right|^{2}-\left|\operatorname{div} \nu_{u, \sigma}\right|^{2}\right)$ is ensured, which is in accordance with the continuous case. In practice, finite differences are used for the numerical evaluation of $\widetilde{\mathcal{B}}_{\sigma}(u)$. Finally, we propose the following algorithm, which converges under the same assumptions as Algorithm 4.1 since the penalty term $\widetilde{\mathcal{B}}_{\sigma}(u)$ is treated explicitly.

Algorithm 4.3. Same as Algorithm 4.1 except that we replace 1) with

1) Using finite differences to evaluate $\widetilde{\mathcal{B}}_{\sigma}\left(u_{k}^{n+1}\right)$, compute $h_{\mathcal{P}}^{n}=u^{n}-\frac{\delta_{t}}{\alpha \varepsilon^{4}} W^{\prime \prime}\left(u_{k}^{n+1}\right) \mu_{k}^{n+1}+\delta_{t} \widetilde{\mathcal{B}}_{\sigma}\left(u^{n}\right)$, and $\tilde{h}_{\mathcal{P}}^{n}=\alpha W^{\prime}\left(u_{k}^{n+1}\right)$.

\subsection{Numerical simulations of the classical flow}

The following simulations have been realized with Matlab. The isolevel sets $\Gamma(t)=\left\{x: u(x, t)=\frac{1}{2}\right\}$ are computed and drawn using the Matlab functions contour in 2D and isosurface in 3D. We use the double-well potential $W(s)=\frac{1}{2} s^{2}(1-s)^{2}$, and we consider the following PDE system with periodic boundary conditions:

$$
\left\{\begin{array}{l}
\partial_{t} u=\Delta \mu-\frac{1}{\varepsilon^{2}} W^{\prime \prime}(u) \mu \\
\mu=\frac{1}{\varepsilon^{2}} W^{\prime}(u)-\Delta u
\end{array}\right.
$$

and with initial conditions $u(x, 0)=\gamma\left(\frac{d(x, E)}{\varepsilon}\right), \mu(x, 0)=-\frac{1}{\varepsilon} \Delta d(x, E) \gamma^{\prime}\left(\frac{d(x, E)}{\varepsilon}\right)$.

We plot on Figure 2 the graph of $t \mapsto u_{\varepsilon}(\cdot, t)$ computed for different values of $\varepsilon$. We choose for the other parameters: $P=2^{7}, \delta_{t}=1 / \mathcal{P}^{-4}$. In the first experiment, obtained with $\varepsilon=5 / \mathcal{P}$, the two circles merge. In contrast, a crossing of interfaces appear for the case $\varepsilon=1.5 / \mathcal{P}$. This corresponds to a solution of the Allen-Cahn equation with unsmooth nodal set. After contact, the interfaces continues to evolve while the crossing seems to be numerically stable and does not influence the interface evolution. More precisely, the interface $\Gamma(t)$ seems to converge to a growing eight, which is one of the closed planar elasticae described in Langer and Singer's work [17].

In Figure 3 , we present experiments which illustrate the formation of singularities in dimension 3 . On the left, two spheres evolve by the classical diffuse flow. As the distance between the two spheres is about $\varepsilon$, they 

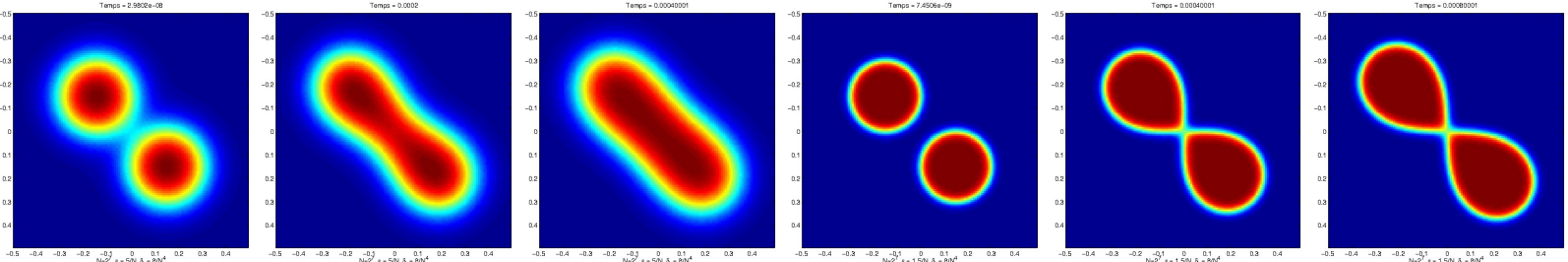

FiguRE 2. Evolution by the classical approximation of the Willmore flow of two disjoint circles, for various values of $\varepsilon$. First three images: $\varepsilon=5 / \mathcal{P}$; Last three images: $\varepsilon=1.5 / \mathcal{P}$; The curve $\Gamma(t)$ is observed at times: $t=0$ (left), $t=0.0004$ (middle), $t=0.0008$ (right).

merge. The second example shows the evolution of a cube cut by a plane (more precisely, both the plane and the cube's boundary separate the two phases. The cube seems to evolve to a sphere without being disturbed by the presence of the plane. All these experiments show that the classical diffuse flow may yield singularities, although the comprehension of singular solutions to the Allen-Cahn equation in dimension 3 remains incomplete.
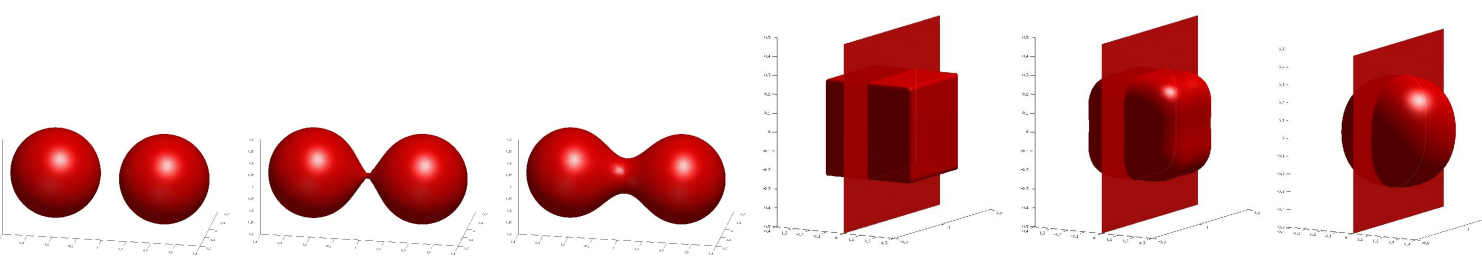

Figure 3. 3D-examples of evolutions by the classical diffuse flow yielding singularities.

\subsection{Numerical simulations of Mugnai's flow}

We now consider the PDE system associated with Mugnai's flow: $\left\{\begin{array}{l}\partial_{t} u=\Delta \mu-\frac{1}{\varepsilon^{2}} W^{\prime \prime}(u) \mu+\widetilde{\mathcal{B}}_{\sigma}(u) \\ \mu=\frac{1}{\varepsilon^{2}} W^{\prime}(u)-\Delta u .\end{array}\right.$, where $\widetilde{\mathcal{B}}_{\sigma}(u)=W^{\prime}(u)\left[\left(\left|\nabla \nu_{u, \sigma}\right|^{2}-\left|\operatorname{div} \nu_{u, \sigma}\right|^{2}\right)-\operatorname{curl}\left(\operatorname{curl}\left(\nu_{u, \sigma}\right)\right) \cdot \nu_{u, \sigma}\right]$ with $\nu_{u, \sigma}=\frac{\nabla u}{\sqrt{|\nabla u|^{2}+\sigma^{2}}}$. The initial conditions are $u(x, 0)=\gamma\left(\frac{d\left(\Gamma_{0}\right)}{\varepsilon}\right)$ and $\mu(x, 0)=-\frac{1}{\varepsilon} \Delta d\left(\Gamma_{0}\right) \gamma^{\prime}\left(\frac{d\left(\Gamma_{0}\right)}{\varepsilon}\right)$. We set the approximation parameter $\sigma=10^{-3}$ and we solve numerically the system using Algorithm 4.3.

We present two experiments in Figure 4 obtained with the set of parameters $\mathcal{P}=2^{7}, \varepsilon=2 / \mathcal{P}$ and $\delta_{t}=$ $\varepsilon^{2} \mathcal{P}^{-2} / 8$. The simulations indicate that the additional penalization term $\widetilde{\mathcal{B}}_{\sigma}(u)$ prevents the interfaces from colliding, in contrast with the classical flow. More precisely:
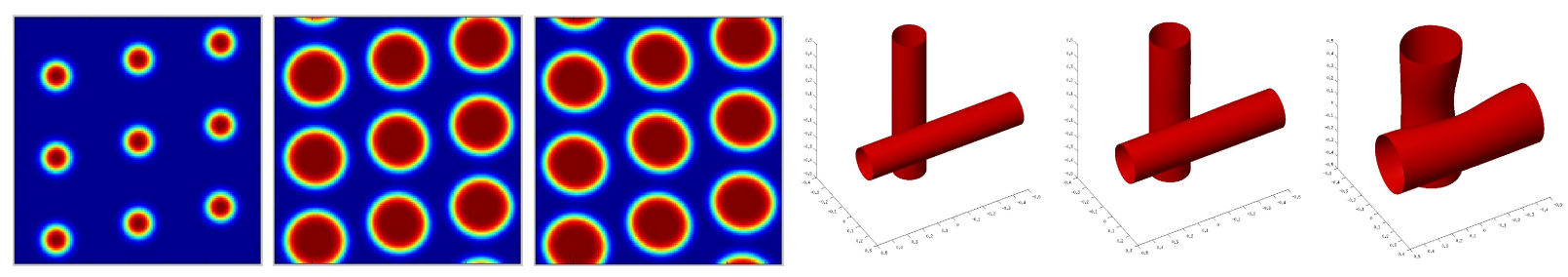

FiguRE 4. Illustrations in 2D and 3D that Mugnai's flow prevents from colliding. 
- As long as the interfaces are smooth, Mugnai's and the classical flow behave in the same way, which was of course expected from the theoretical properties of the associated functionals. In particular, the penalization term $\widetilde{\mathcal{B}}_{\sigma}(u)$ has no critical influence on the evolution of a smooth interface, as long as the evolution remains smooth as well with the classical flow.

- Since Mugnai's energy $\mathcal{W}_{\varepsilon}^{\mathrm{Mu}} \Gamma$-converges in dimension 2 to the relaxation of the Willmore energy, the associated flow prevents from crossing, which is confirmed by the simulations. In 3D as well, our simulations indicate that no crossing should occur. This indicates that the $\Gamma$-convergence property should also be true in 3D for Mugnai's energy, which is so far an open question that requires a better understanding of the diffuse approximation of the genus (having in mind the Gauss-Bonnet Theorem).

\section{REFERENCES}

[1] G. Bellettini. Variational approximation of functionals with curvatures and related properties. J. Convex Anal., 4(1):91-108, 1997.

[2] G. Bellettini, G. D. Maso, and M. Paolini. Semicontinuity and relaxation properties of curvature depending functional in 2D. Annali della Scuola Normale di Pisa, Classe di Scienze, $4^{e}$ série, 20(2):247-297, 1993.

[3] G. Bellettini and L. Mugnai. On the approximation of the elastica functional in radial symmetry. Calc. Var. Partial Differential Equations, 24:1-20, 2005.

[4] G. Bellettini and L. Mugnai. Approximation of Helfrich's functional via diffuse interfaces. SIAM J. Math. Anal., 42(6):24022433, 2010.

[5] G. Bellettini and M. Paolini. Approssimazione variazionale di funzionali con curvatura. In Seminario Analisi Matematica, Univ. Bologna, pages 87-97, 1993.

[6] G. Bellettini and M. Paolini. Quasi-optimal error estimates for the mean curvature flow with a forcing term. Differential Integral Equations, 8(4):735-752, 1995.

[7] E. Bretin, S. Masnou, and E. Oudet. Phase-field approximations of the Willmore functional and flow. Technical report, 2013.

[8] G. Caginalp and P. C. Fife. Dynamics of layered interfaces arising from phase boundaries. SIAM J. Appl. Math., 48(3):506-518, 1988.

[9] P. Colli and P. Laurencot. A phase-field approximation of the Willmore flow with volume constraint. Interfaces Free Bound, 13:341-351, 2011.

[10] P. Colli and P. Laurencot. A phase-field approximation of the Willmore flow with volume and area constraints. SIAM J. Math. Anal., 44:3734-3754, 2012.

[11] H. Dang, P. C. Fife, and L. Peletier. Saddle solutions of the bistable diffusion equation. Zeitschrift fur angewandte Mathematik und Physik ZAMP, 43(6):984-998, Nov. 1992.

[12] E. De Giorgi. Some remarks on $\Gamma$-convergence and least square methods. In G. D. Maso and G. Dell'Antonio, editors, Composite Media and Homogenization Theory, pages 135-142. Birkhaüser, Boston, 1991.

[13] G. Dziuk, E. Kuwert, and R. Schätzle. Evolution of elastic curves in $\mathbb{R}^{n}$ : Existence and computation. SIAM J. Math. Anal., 33(5):1228-1245, 2002.

[14] S. Esedoḡlu, A. Rätz, and M. Röger. Colliding Interfaces in Old and New Diffuse-interface Approximations of Willmore-flow. ArXiv e-prints, Sept. 2012.

[15] E. Kuwert and R. Schätzle. Removability of point singularities of Willmore surfaces. Ann. of Math. (2), 160(1):315-357, 2004.

[16] E. K. Kuwert and R. Schätzle. The Willmore flow with small initial energy. Differential Geom, 57(3):409-441, 2001.

[17] J. Langer and D. A. Singer. Curve straightening and a minimax argument for closed elastic curves. Topology, 24:75-88, 1985.

[18] P. Loreti and R. March. Propagation of fronts in a nonlinear fourth order equation. European Journal of Applied Mathematics, 11:203-213, 32000.

[19] U. F. Mayer and G. Simonett. A numerical scheme for axisymmetric solutions of curvature driven free boundary problems, with applications to the Willmore flow. Interfaces Free Bound, 4(1):89-109, 2002.

[20] R. Moser. A higher order asymptotic problem related to phase transitions. SIAM J. Math. Analysis, 37(3):712-736, 2005.

[21] L. Mugnai. Gamma-convergence results for phase-field approximations of the 2D-Euler elastica functional. ArXiv e-prints, Sept. 2010.

[22] Y. Nagase and Y. Tonegawa. A singular perturbation problem with integral curvature bound. Hiroshima Mathematical Journal, $37: 455-489,2007$.

[23] R. L. Pego. Front migration in the nonlinear Cahn-Hilliard equation. Proc. Roy. Soc. London Ser. A, 422(1863):261-278, 1989.

[24] M. Röger and R. Schätzle. On a modified conjecture of De Giorgi. Math. Z., 254(4):675-714, 2006.

[25] S. Serfaty. Gamma-convergence of gradient flows on hilbert and metric spaces and applications. Disc. Cont. Dyn. Systems, 31(4):1427-1451, 2011.

[26] Y. Tonegawa. Phase field model with a variable chemical potential. Proceedings of the Royal Society of Edinburgh: Section A Mathematics, 132:993-1019, 72002. 